\title{
ZUSAMMENFASSUNG UND AUSBLICK: METZ - EINE DEUTSCHE STADT?
}

An zwei großen Belagerungen bricht sich in neuerer Zeit die Geschichte von Metz. Innerhalb dieser Zeitspanne, also zwischen 1552 und 1870, entwickelt sich die Stadt zum Militärstandort, dem Grenznähe und Garnison das besondere Gepräge verleihen. Neben dem Festungscharakter bleibt Metz ein bedeutender Wirtschaftsstandort und das traditionell auch politisch mächtige religiöse Zentrum. Von dynastischen in nationale Spannungsfelder geratend, wird der Platz seit Ausgang des 17. Jahrhunderts zur Schlüsselbastion eines sich zwischen Frankreich und Deutschland herausbildenden Grenzlandes. Die über weite Strecken ruhige Entwicklung der Stadt selbst korrespondiert mit einem für das Umland stets problematischen Wechselverhältnis von Soldaten und Zivilisten. Dabei können fremde Heere ebenso wie die in Metz stationierte Truppe zum Alptraum für Land und Leute werden. Die Belagerung von 1870 steht hierfür als extremes Beispiel. Mit der Kapitulation der letzten kaiserlichen Armee unter Marschall Bazaine am 27. Oktober 1870 in Metz ist zugleich die Niederlage des napoleonischen Frankreichs besiegelt. Nach fünf großen Schlachten ${ }^{1}$, zahlreichen kleineren Gefechten und einer fast zehnwöchigen Belagerung hatte sich im Spätsommer und Herbst 1870 das Schicksal der alten Reichsstadt und ihrer Bewohner entschieden, die schöne Gegend um die Festung ein Blutbad durchlitten, wie es seit dem Hunnensturm und der Schlacht auf den Katalaunischen Feldern nicht mehr dagewesen war. Metz, so nimmt es sich aus heutiger Sicht aus, ist das Verdun von 1870. Auch das Ausgreifen des Festungskrieges auf die Zivilbevölkerung, die starke Sogwirkung des Waffenplatzes auf das Umland deutet in Ansätzen auf Muster des totalen Krieges ${ }^{2}$ hin. Die Verluste waren auf keinem Schlachtfeld des Deutsch-Französischen Krieges höher als hier, wo weit über 60000 Menschen den Tod fanden oder verwundet wurden. Die in der Gefangenschaft verstorbenen Rheinarmisten fallen noch zusätzlich ins Gewicht. Hatten die Schlachten vom 16. und 18. August noch keine endgültige Entscheidung herbeigeführt, so waren mit der Übergabe der Festung die Würfel gefallen. Der Krieg trat in seine zweite Phase ein, in der die deutschen Bundestruppen nun gegen die Heere der Republik zu Felde zogen. Gleichsam Symbol und Bedingung für den Zusammenbruch des französischen Kaisertums und die nachfolgende Unterwerfung des Landes, bedeutete Metz auch einen grundlegenden Schritt auf dem Weg zur deutschen Reichsgründung und zur Kaiserproklamation Wilhelms I. in Versailles. Sedan wurde zur Entscheidungs-

1 Colombey, Mars-la-Tour, Gravelotte, Noisseville und Woippy.

2 FÖRSTER, NAGLER, On the road. 
schlacht in politischer Hinsicht, Metz hingegen, das ungleich schwerer umkämpft war, brachte die Deutschen militärisch auf die Siegerstraße.

Als alte Garnisonsstadt an der „künstlichen und gefährlichsten Grenze Frankreichs ${ }^{\text {*3 }}$ war Metz nicht zufällig zum wichtigsten Kriegsschauplatz von 1870 geworden. Seit Jahrhunderten Verkehrsknotenpunkt, Wirtschaftsfaktor und administratives Zentrum, hatten Könige und Kaiser ihre Hände nach der Stadt ausgestreckt, aber auch lange Friedenszeiten eine fruchtbare Entwicklung befördert. Mit Vaubans Neuerungen begann ihr Aufstieg zu einem nicht nur militärischen, sondern kriegerischen Platz, der nicht bloße Garnison, sondern eben strategischer Schlüssel für den politischen Vorrang auf dem Kontinent sein sollte. Daß Metz in eine größere Auseinandersetzung zwischen Deutschland und Frankreich hineingeraten würde, schien unausweichlich, doch gab es eine Identität des Ortes unterhalb und jenseits nationaler Grenzen.

Der Belagerungsalltag von 1870 verlief für Soldaten wie Zivilisten gleichermaßen an "den Grenzen des Seins“. Während die Deutschen unter den Folgen der Schlachten von Mars-la-Tour und Gravelotte, an den Witterungsunbilden, einem erheblichen Ausrüstungsmangel und allgemeiner Quartiernot litten, zwang die in die Stadt gedrängte Rheinarmee letztlich der Hunger in die Knie. Ruhr und Typhus breiteten sich beiderseits der Belagerungslinien aus und konnten durch die mangelnde medizinische Betreuung nicht bekämpft werden. Tausende von Soldaten und Zivilisten starben. Jämmerlich, so konnte gezeigt werden, sah die Lage der Verwundeten aus, deren Überlebenschance gering war. Gleichwohl haben wir neben dem Stellungskrieg um die Festung auch im medizinischen Bereich wie in Infrastruktur und Mobilität zumindest in Ansätzen die Züge des modernen Krieges zu erkennen. Während sich die Eingeschlossenen nahezu hermetisch von Restfrankreich abgeriegelt sahen, beunruhigten im Hinterland Freischärlerverbände, Franctireurs genannt, die Besatzer und versuchten, in Kontakt mit der blockierten Stadt zu kommen und den Belagerungsring aufzuweichen. Die Erfahrung derartiger, oft erbittert geführter Auseinandersetzungen wie ihre propagandistische Aufbereitung ließ beiderseits Feindbilder manifest werden und nationales Empfinden wachsen. Eine Brutalisierung und Militarisierung des gesellschaftlichen Wertekosmos geht für die deutsche Seite damit einher. Gleichzeitig lieferte der Belagerungskrieg um Metz Beispiele der Achtung und Ehrenbezeugung gegenüber dem Feind und seinen Führern. Die korrekte Behandlung deutscher Gefangener in der Festung sowie die vielen anerkennenden Äußerungen gegenüber der geschlagenen Rheinarmee stehen dafür; die Überführung nach und die Betreuung der riesigen französischen Gefangenenheere in Deutschland sowieso.

Auch die Situation der Belagerungstruppen war schwierig. Schlecht informiert, führten sie ein Maulwurfsdasein in vieler Hinsicht, hausten in Erd-

${ }^{3}$ BRAUdel, Frankreich, Teil I, S. 338. 
und Holzhütten oder lagen unter freiem Himmel. Die rückwärtigen Verbindungen in die Heimat genügten den Anforderungen in keiner Weise, und Streitigkeiten zwischen den Korpsführungen verübelten den Soldaten das ohnedies triste Vorpostendasein zusätzlich. Der großen Langeweile wurde durch abenteuerliche Patrouillengänge und kleine Scharmützel begegnet. Friedliche Kontakte zwischen Franzosen und Deutschen gehörten ebenfalls ins Bild, ergaben sich während längerer Einquartierungen oder dem Wachdienst im Niemandsland, wo sich die Vorposten begegneten und die Ärmsten nach Kartoffeln suchen ließen. Die Stellungskämpfe des Ersten Weltkrieges deuten sich in manchem an: „Vor Metz nichts Neues“ hätte für die Zeit nach dem gescheiterten großen Ausfall vom 1. September öfter vermeldet werden können. Während aber die Ereignisse stillzustehen schienen, wurden Identitäten um- und ausgeprägt. Was die Leute trotz menschenunwürdiger Lage aufrecht hielt, waren der Glaube an Gott und der briefliche Kontakt zu den Angehörigen - eine Dreiheit von Religion, Vaterland und Familie, wie man es besonders für die deutsche Seite hervorgehoben hat ${ }^{4}$. Für Hans von Kretschman, den preußischen Offizier, konnte dies gezeigt werden.

Die Kapitulation der Rheinarmee beendete nicht nur das Leiden der entkräfteten und ausgehungerten Franzosen, sondern kam auch für die Belagerer einem Glücksumstand gleich. Im Fall einer ausreichenden Verproviantierung und des Widerstands der Festung über den Winter hätte diesen, in erster Linie wegen der fehlenden festen Unterkünfte, mit hoher Wahrscheinlichkeit ein Fiasko bevorgestanden. Durch die Kapitulation gerieten 170000 Franzosen in deutsche Kriegsgefangenschaft. Die Niederlage Frankreichs zeichnete sich ab. Die Gründung des deutschen Kaiserreiches am 18. Januar 1871 und die Kapitulation von Paris wenige Tage später beendeten den Krieg. Im Frankfurter Friedensvertrag mußte Frankreich das Elsaß und einen großen Teil Lothringens abtreten. Hinzu kamen 1,5 Milliarden Taler Kriegsentschädigung. Metz wurde westlicher Vorposten Deutschlands und damit Pfahl im Fleische Frankreichs. Im Chor der Jubelgesänge gingen warnende Stimmen unter, die „saure Stunden“ prophezeiten, um das Erreichte zu behaupten.

In Deutschland hatte die öffentliche Meinung lange vor Kriegsende über die Zukunft Elsaß-Lothringens entschieden. Zu Anfang, wie früher ausgeführt, noch wenig annexionistisch gestimmt, dabei nur gelegentlich an die einstige Zugehörigkeit des Grenzlandes zum Reich erinnernd, war man bald davon überzeugt, daß es Frankreich weggenommen werden müsse ${ }^{5}$. Daß mit Blick auf die deutschen Denkhaushalte der Zeit anstatt von „Annexion“ besser von „Rückforderung", „Rücknahme“ oder „Wiedergewinnung" gesprochen werden sollte, hat Bronner nicht ganz zu Unrecht angemerkt ${ }^{6}$. Unüblich waren Grenzverschiebungen nach Kriegen ja keineswegs. So schrieb

4 Vgl. MONOD, Allemands et Français, S. 66.

${ }^{5} \mathrm{KOLB}$, Weg aus dem Krieg, 125.

6 Bronner, 1870/71, Bd. 1, S. 24. 
etwa die englische Times bereits kurz nach Sedan, daß die Annexion Deutsch-Lothringens mit Metz die niedrigste aller legitimen deutschen Forderungen sei, und die Daily News sprachen von einer "natürlichen Strafe “ für Frankreich ${ }^{7}$. Andererseits wußten nicht nur linke Kritiker, daß derartige Grenzveränderungen doch nur Provisorien blieben und nicht „für die Ewigkeit $^{\text {“ }}$ gemacht waren ${ }^{8}$.

Mit dem Frieden wurde Metz Hauptstadt des Bezirks Lothringen im Reichsland Elsaß-Lothringen. Noch bei den Verhandlungen über den Vorfrieden Ende Februar 1871 hatte Thiers zwar der Abtretung des Elsaß mit Straßburg zugestimmt, Lothringen und Metz dagegen hartnäckig verweigert. Und zweifellos stand das Elsaß dem Reich näher als Metz und Lothringen, wo literarisch-wissenschaftliche Berührungspunkte, die vor allem Straßburg an den Oberrhein banden, vollkommen fehlten ${ }^{9}$. Selbst Bismarck wäre ohne den Druck Moltkes und der Militärs hier vielleicht zum Einlenken bereit gewesen, denn er wußte, daß man damit Frankreichs Ehrgefühl schwer verletzte. Bismarcks Auffassung in der Sache spiegelt ein Brief Wilhelms I. an die französische Exkaiserin Eugénie vom 26. Oktober 1870, also unmittelbar vor der Kapitulation der Rheinarmee. Hier ist von "Sicherheit" und Vorbereitung auf den nächsten Krieg die Rede und eben auch von notwendigen „Gebietsabtretungen [...], die kein anderes Ziel haben, als die Ausgangsstellungen der französischen Armeen zurückzudrängen, die uns in Zukunft angreifen werden"10. Diese Formulierung macht auch klar, daß der Krieg im Bewußtsein der Zeit keine irreversiblen Zustände schaffen konnte und wollte und somit auch nicht als ein letztgültiger Existenz- oder Daseinskampf begriffen wurde. Vielmehr glaubte man an ein ewig wiederkehrendes Auf und $\mathrm{Ab}$ gleich einem Spiel, das keine absoluten Sieger kannte. Clemenceau übrigens soll eben jenes Schreiben bei der Aushandlung der Versailler Friedensbedingungen den Amerikanern vorgelegt haben, um seinen Anspruch auf Elsaß-Lothringen zu bekräftigen ${ }^{11}$.

Der Streit darum, wie deutsch oder französisch die Stadt Metz nun wirklich war, setzte bald nach dem Einzug der Deutschen ein und entbehrte dann nicht selten fundierter sachlicher Grundlagen ${ }^{12}$. Indes mußten auch die eifrigsten Propagandisten des Deutschtums anerkennen, daß die französischen Elemente über die Jahrhunderte gestärkt worden und den deutschen inzwischen weit überlegen waren. Metz, von jeher romanisch kultiviert und jenseits der deutschen Sprachgrenze gelegen, hatte sich anders als Straßburg zu

\footnotetext{
7 Zit. nach FIDELAK, Sedan, S. $47 \mathrm{f}$.

$8 \mathrm{Vgl}$. BRONNER, 1870/71, Bd. 1, S. 125-142.

9 WOLFRAM, Wissenschaft, S. $76 \mathrm{f}$.

10 Zit. nach BRONNER, 1970/71, Bd. 2, S. 497.

11 Ibid.

12 Vgl. Westphal, Metz, Teil I, Vorwort.
} 
einer durch und durch französischen Stadt entwickelt ${ }^{13}$, die man nun dem Reich zuschlug. Kompromißvorschläge, die auf die Mosellinie ohne Metz zielten, für den Fall, daß die Festung geschliffen würde ${ }^{14}$, setzten sich nicht durch. Letztlich blieb auch für Bismarck der Schutz der süddeutschen Grenze das zentrale Motiv für die Annexion ${ }^{15}$. Aus nationaler Perspektive der Nachkriegszeit nahmen sich die Dinge nun so aus: Durch den Verlust des Elsaß hatte man die Franzosen gezwungen, hinter die Zeiten Ludwigs XIV., mit der Abtretung von Metz sogar hinter jene Heinrichs II. zurückzugehen ${ }^{16}$. Der „Politik Richelieus“" war ein Ende gesetzt. Dies bedeutete zugleich, die verheerenden Wirkungen des Belagerungskrieges von 1870 für Metz und Lothringen als einen fast zwangsläufigen und legitimen historischen Reflex auf die Verwüstung der Pfalz von 1688/89 durch die Franzosen zu verstehen.

Für Frankreich tritt an die Stelle des verlorenen Waffenplatzes fortan Verdun. Nach und nach wird dieses nun zur stärksten Festung innerhalb eines neuen Gürtels ausgebaut, der sich halbkreisförmig von Reims über Toul und Nancy bis Épinal und Belfort zieht. Letzteres behält man gegen die Abgabe eines französisch-lothringischen Landstriches ein ${ }^{17}$. Metz hingegen entwikkelt sich neben Berlin mit bald 25000 Mann Besatzung zur stärksten Garnison im Kaiserreich. ${ }^{18}$ Es wird fortifikatorisch und waffentechnisch noch weiter verstärkt und um 1890 als Ausgangspunkt für eine nun wieder ernsthafter kalkulierte deutsche Westoffensive der Zukunft betrachtet. Ein zweiter äußerer Kranz von starken Panzerfestungen entsteht, dazu bombensichere Unterstände und Gefrieranstalten für den Belagerungsfall. Auch ein neues Lazarett wird errichtet ${ }^{19}$. Häufig finden jetzt Manöver statt, bei denen der Kaiser als Inhaber der höchsten Gewalt im Reichsland gern selbst den Oberbefehl übernimmt ${ }^{20}$. Der Dienst bei den dort stationierten und durch die Vorgeschichte besonders renommierten Kavallerieeinheiten gilt als besondere Schinderei. Man kann dies aus Franz Rehbeins Leben eines Landarbeiters von 1911 sehr schön ersehen ${ }^{21}$. Aber auch die Franzosen machen entlang der

13 Vor Kriegsbeginn lebten 1741 Deutsche in der Stadt. Folz, Metz als deutsche Bezirkshauptstadt, S. 372.

14 KRETSCHMAN, Kriegsbriefe, S. 346. Auch für die städtische Infrastruktur wäre dies eine durchaus günstige Lösung gewesen.

15 KOLB, Weg aus dem Krieg, S. 157. PARISSE, Lothringen, S. $409 \mathrm{f}$. Interessant die vor allem von Walter Lipgens und Lothar Gall geführten Debatten in der Historischen Zeitschrift Ende der 60er Jahre, die sich im Kern weniger um Metz und die Frage der Annexion als um die Beurteilung Bismarcks drehten. Vgl. HZ 206 (1968) S. 265-326; 586-617.

16 Cartellieri, Deutschland und Frankreich, S. 14.

17 Für Belfort opferte man die Dörfer des Oberlandes (Pays-Haut). Vgl. Parisse, Lothringen, S. 410.

18 KEUNE, Erinnerung, S. 16. LANGSDORF, Metz als Militärstadt, S. 545.

19 Ibid S. 542.

20 PARISSE, Lothringen, S. 418.

21 Auszug in: RITTER, KOCKA, Deutsche Sozialgeschichte, S. 237-240. 
verlorenen Gebiete immer wieder mobil. Lothringen starrt beiderseits der neuen Grenze vor Waffen und Truppen, was den durch den Krieg noch verschärften deutsch-französischen Gegensatz widerspiegelt. Es liegt so bereits auf der Hand, daß diese Massierung im Falle eines künftigen Krieges schnelle Durchbrüche kaum mehr zulassen würde. Der Schlieffen-Plan, der einen deutschen Vormarsch durch Belgien und Nord-Ost-Frankreich vorsieht, ist die höchst problematische Antwort auf diese Konstellation. Militärische Prämissen engen den politischen Handlungsspielraum fortan mehr und mehr ein und zwingen im Falle eines Konflikts England, das die belgische Neutralität schon 1870 zur Gretchenfrage seiner Außenpolitik gemacht hatte, fast notwendig auf die Seite Frankreichs 22. Um 1910 ist der Landstrich von allen Gegenden der Erde derjenige mit der höchsten Konzentration an Soldaten und Kriegstechnik ${ }^{23}$. So wie der Fall von Metz die Niederlage der Franzosen 1870/71 versinnbildlicht, so wird ihr Standhalten vor Verdun zum Faustpfand ihres Sieges über Deutschland im Ersten Weltkrieg. Frankreich jedenfalls, so scheint es im Nachhinein, hatte aus seinen Fehlern besser gelernt als die scheinbar makellos dastehenden Sieger. Mit dem Versailler Friedensvertrag von 1919 wurde der Status quo ante wieder hergestellt, von 1940 bis 1944 durch die deutsche Besetzung faktisch noch einmal aufgehoben.

Noch im November 1870 begann man in Metz mit dem Austausch der Straßenschilder und der Neuaufstellung von Denkmälern. Neben Faberts und Neys Standbilder, die unangetastet blieben, traten die bronzenen Reiterfiguren Kaiser Wilhelm I. und des Kronprinzen. Dem "Sieger von Metz“ Prinz Friedrich Karl wurde ebenfalls ein prächtiges Denkmal gesetzt, und das Tor, durch das er in die Festung eingezogen war, nach ihm benannt. Im November 1918 haben es dann wiederum die Franzosen eilig, diese Denkmäler zu schleifen, halten dies sogar minutiös im Foto fest ${ }^{24}$. Es ist das, was mit nationaler Erziehung umschrieben werden kann. Die Forts, deren östliche Wallungen Wilhelm II. später abtragen ließ, um einer dringend notwendigen Stadterweiterung zu Zwecken des Wohnungsbaus Raum zu schaffen ${ }^{25}$, erhielten Namen von Heerführern wie Alvensleben, Voigts-Rhetz, Manteuffel oder Steinmetz, der damit - wir hatten von seinen Verfehlungen gehört - offenbar rehabilitiert werden sollte. Auch wenn all diese raschen Maßnahmen zunächst nicht mehr als eine Umetikettierung waren: „Deutsches Wesen“ sollte den Platz neu oder wieder beseelen, und mit Treitschke glaubte man am besten zu wissen, was eine Region braucht, der man auch „wider ihren Willen ihr eigenes Selbst zurückgeben“" wollte ${ }^{26}$. Allein im Anliegen problematisch,

22 Zum Hasardspiel des Schlieffen-Planes überzeugend WEHLER, Gesellschaftsgeschichte, S. 1114-1120.

23 PARISSE, Lothringen, S. $418 \mathrm{f}$.

${ }^{24}$ Auf dem umgestürzten Reiterstandbild Friedrichs III. läßt man etwa drei Metzer Damen posieren. Vgl. MuteleT, Metz, S. 123-125.

25 WITTENBROCK, Stadterweiterung, S. 1-23.

26 TREITSCHKE, Was fordern wir, S. 389. 
waren deutsches Militär und deutsche Verwaltung, die nun Einzug hielten und das Stadtbild für die nächsten fast fünfzig Jahre prägen sollten, dazu allein freilich kaum in der Lage. Metz verlor trotz Friedensschluß und Abtretung seinen französischen Charakter nicht, und „die Germanisierung“, wie ein Kundiger im März 1871 voraussah, „wird wohl noch einige Umstände machen" 27 .

Auch der erwähnte „Totenkult“, der als gezielter Erinnerungskult und eben nationale Erziehungsarbeit zur Stärkung der deutschen Identität seit den 90er Jahren vor allem um Gravelotte organisiert wurde ${ }^{28}$, konnte daran nichts Entscheidendes ändern. Zwar erlangen Deutsche, anders als im übrigen Reichsland, schon bald „numerische Überlegenheit" - bis 1875 wandern derer $14000 \mathrm{zu}$, während nach und nach über 20000 Franzosen, darunter vor allem Begüterte und die Masse der Intelligenz, die Stadt vornehmlich in Richtung Nancy verlassen ${ }^{29}$. Doch hält sich „französisches Wesen“ im Kleinen. Die neue Amtssprache gilt lange nur für Garnison und Administration. Kein einheimischer Bäcker oder Fleischer kommt auf die Idee, deutsch zu sprechen, und mancher von Berlin abgeordnete Beamte hätte sich anfangs ohne seine französischen Dienstboten Konserven aus Saarbrücken kommen lassen müssen, um zu überleben. Fontane berichtet vom Streit mit dem Pförtner eines Gasthofes, weil dieser nicht deutsch sprechen wollte und, wie sich dann herausstellte, auch gar nicht konnte ${ }^{30}$. Später mehren sich deutsche Händler proportional zur wachsenden Garnisonsstärke. Das Militär bleibt der dominierende Faktor, und erst nach 1900 gelingt es der städtischen Verwaltung, der Festungsgarnison langsam und für erhebliche Summen Bauland abzuringen ${ }^{31}$. 188 Neubauten entstehen so bis 1913 auf und vor dem Gelände der niedergelegten Wälle; hinzu kommen neue Wasserleitungen und Straßen als Bedingung für die Eingemeindung einer Reihe von Vororten ${ }^{32}$. Der eindringende „deutsche Habitus" kann dabei an Traditionen des französischen Militarismus anknüpfen und verstärkt so bereits vorherrschende „Modelle des Befehlens und Gehorchens" gegenüber jenen noch für das Metz des 17. Jahrhunderts typischen des „Verhandelns und Überredens" weiter ${ }^{33}$. Inso-

27 Der Generalstabsoffizier Paul Bronsart von Schellendorf hatte im Frühjahr zusammen mit Moltke Elsaß-Lothringen bereist und dabei festgestellt, daß Metz eine „rein französische Stadt" sei. BRONSART, Kriegstagebuch, S. 385 f.

28 MAAS, Politische Ikonographie, S. 197.

29 Die für Frankreich Optierenden mußten bis zum 1. Oktober 1872 die Stadt verlassen haben. Nach und nach stieg der deutsche Bevölkerungsanteil in Metz auf über 60\%, wogegen die durchschnittliche Quote im übrigen Elsaß-Lothringen bei weniger als $20 \%$ lag. Histoire de la population, S. 215.

$30 \mathrm{Vgl.} \mathrm{BRONNER,} \mathrm{1870/71,} \mathrm{Bd.} \mathrm{1,} \mathrm{S.} 98$.

31 WITTEnBRock, Stadterweiterung, S. 11-16. Folz, Metz als deutsche Bezirkshauptstadt, S. 377.

32 Ibid S. 378-381.

33 Norbert Elias' Gegenüberstellung dieser beiden Prinzipien als jeweilige Grundzüge differierender nationaler Charakterbildungen (hier für Holland und Deutschland) sind 
fern bleibt die Stadt mit ihrem spezifischen Charakter eine deutsch-französische Schöpfung, ein Ort also, dessen grenzübergreifende Geltung nach und nach durch nationalistische Abschottung und Instrumentalisierung verloren geht.

Ein tiefer gehender kultureller Wandel zugunsten des Deutschtums griff trotz oder gerade wegen der überragenden militärischen Präsenz nicht, zumal der Widerspruch zwischen preußischem Protestantismus und reichsländischem Katholizismus die Dinge zusätzlich störte ${ }^{34}$. Bischof Dupont des Loges war hier treibende Kraft einer profranzösischen Stimmung, in der katholische mit nationalistisch-chauvinistischen Positionen verschmolzen ${ }^{35}$. Auch auf der politischen Ebene verteidigten Frankreich-Anhänger und „Autonomisten" das Feld und machten im Reichstag geschlossen Front gegen Deutschland. Im übrigen schürte gerade die große Emigrantenschar von $\mathrm{Pa}$ ris und Nancy her den Geist der Revanche ${ }^{36}$. Aus der alten Herzogsstadt pulste nun eine lothringische Bewegung, die auf den deutschen Teil ausstrahlte, den abzuschreiben sie niemals gewillt war. Neben der Lyrik Guérins oder Paul Verlaines, deren melancholische Texte der französischen Zeit nachtrauerten, verwerteten Maurice Barrès' Romane Au service de l'Allemagne oder Colette Boudoche die Grenzgegend und das Metzer Land auch literarisch. In Metz, so ist hier nachzulesen, zeige sich der deutsch-französische Dualismus besonders deutlich in der Kluft zwischen den neu entstehenden deutschen Bierhallen und einer tradierten, weiterhin exklusiv französischen Kaffeehauskultur. Mit dem Bier, so meinte Barrès, begann sich „l'ennui teuton “, die deutsche Langeweile, des Ortes zu bemächtigen ${ }^{37}$. Franzosen trafen sich jetzt zur Erinnerung der Kämpfe von 1870 alljährlich in Mars-la-Tour nahe der neuen Grenze. Auch viele Metzer mischten sich da unter die Kundgebungsteilnehmer, „um einen Tag lang Franzosen in Frankreich zu sein“ und den patriotischen Predigten des „Bischofs von der Grenze“ Mgr. Turi$n^{n} z^{38}$ zu folgen. Vor allem der Nationalfeiertag Frankreichs, an dem man in Grenznähe große Militärparaden abhielt, beseelte die Annektierten, selbst wenn sie nur Zaungäste blieben, mit der Hoffnung auf Rückkehr und Revanche ${ }^{39}$. Die zahlreichen Dorffeste auf französischem Territorium boten zudem

für Metz eher im Neben- und Nacheinander unter französischem und deutschem Einfluß auszumachen. Vgl. Elias, Studien, S. $19 f$.

${ }^{34}$ Der elsaß-lothringische Katholizismus entpuppte sich besonders in der Phase des Kulturkampfes als hartnäckiger Widerstandsherd gegen jede Verpreußung. Der katholische Bevölkerungsanteil betrug 1871 rund $82 \%, 1910$ noch rund $76 \%$. HALLIER, Kirche und Schule, S. 106.

35 STÄHLIN, Geschichte Elsaß-Lothringens, S. $216 \mathrm{f}$.

36 Ibid.

37 Zit. nach Mutelet, Metz, S. 12.

38 PARISSE, Lothringen, S. 415. Hierher die Aufstellung und Segnung eines gebrochenen „Lothringer Kreuzes“ mit der Inschrift „Ce nato po tojo“ (Nicht für immer) durch Annektierte. MAAS, A l'extrême frontière, S. 55.

39 Ibid S. $65 \mathrm{f}$. 
einen willkommenen Anlaß, „pour se trouver un moment sous le drapeau français et ,boire du bon vin de France $[\ldots]^{\star 40}$.

Zäher Widerstand gegen die Einführung des Deutschen und die Zurückdrängung des politischen Katholizismus hielt sich im Schulwesen des einverleibten Lothringens, wenngleich aus Metz selbst das Gros der höheren Lehrerschaft samt Schülern abgewandert war. Das dortige Lyzeum entwickelte sich daher in den Augen der französischen Stadtbevölkerung zu einer deutschen Bildungsenklave ${ }^{41}$, der, ähnlich der aufgeblähten Garnison, der Geruch des Fremden wie eines künstlichen Implantats anhaftete. Die Etablierung eines deutschen Theaters in der Stadt mit zunächst deutsch-französischer Truppe und später 32 französischen Pflichtvorstellungen innerhalb eines deutschen Spielplanes war mühselig und gelang nur in Ansätzen. Die Garnisonsträgheit sowie die vielen Ressentiments des französischen Publikums gegenüber den neuen Machern und deren Themen standen dagegen ${ }^{42}$. Auch das Konzertwesen blieb über lange Jahre Angelegenheit der deutschen Militärkapellen, und die Konzerte fanden fast ausschließlich im Militärkasino statt. Bezeichnenderweise schloß man die Franzosen davon bis 1895 aus, wohl wissend, daß deren Nationalhymne so oder so die Marseillaise bleiben würde. Politisch ausgleichend wirkte dagegen ein neu gegründeter Konzertverband, dem nach und nach auch einheimische Kreise beitraten ${ }^{43}$. Von einer Integration der erworbenen Gebiete samt der Mehrheit ihrer Bevölkerung im Reich, einem neuen "Selbst“ der Region also, konnte letztlich keine Rede sein, von „ethnischen Säuberungen“ allerdings auch nicht.

Zunächst nach dem Muster einer preußischen Provinz verwaltet, wurde Elsaß-Lothringen erst 1879 eine eigene Landesregierung zugebilligt, die freilich einem kaiserlichen Statthalter unterstellt blieb. Daß dieser Posten einem Militär, dem Feldmarschall Erwin von Manteuffel, zukam, symbolisiert die weiterhin vorherrschenden Gewichtsverhältnisse. In politischer und sozialer Hinsicht den übrigen Gebieten des Reiches wie ein Wurmfortsatz angehängt, zählte im Grunde lediglich der militärstrategisch bedeutsame Zugewinn und hier besonders das waffenklirrende Metz als wichtigster Brückenkopf. Clemens von Delbrücks Verfassungswerk von 1911 war ein zu spät unternommener Schritt nach vorn, der den Franzosen freilich nicht weit genug ging

40 Ibid S. 67.

41 Hier wurde der deutsche Einfluß bald fühlbar: Bereits 1876 saßen auf den Schulbänken des Metzer Gymnasiums 357 Söhne von Eingewanderten neben 113 Einheimischen. HaLLIER, Kirche und Schule, S. 117. Bronner hat dagegen den deutlichen Überhang einheimischer Lehrer und Schüler in den Volks- und Mittelschulen Elsaß-Lothringens herausgestrichen. DERS., 1870/71, S. $400 \mathrm{f}$. Vgl. auch ARETIN, Erziehung zum Hurrapatrioten, S. 94.

42 NeUfFer, D. u. H., Das Metzer Stadttheater von 1871-1918. In: Reichsland, Bd. III, S. 361-364.

43 WOLFRAM, Wissenschaft, S. 100. 
und von der deutschen Rechten abgelehnt wurde ${ }^{44}$. Über die Qualität der neuen Beamtenschaft zu urteilen, ist müßig, doch drängen sich Vergleiche zum Anschluß der neuen Länder im deutschen Vereinigungsprozeß nach 1989/90 auf. Ein Einheimischer jedenfalls bemerkte damals ganz boshaft, „daß die neuen Beamten in Elsaß-Lothringen den Auswurf der deutschen Nation darstellten “45. In Straßburg verliefen die Dinge anders als in Metz, und vor allem symbolisch kam der neuen Hauptstadt des Reichslandes eine national sinnstiftende Bedeutung zu. Neben dem Exodus der einheimischen Intelligenz und großer Teile des Mittelstandes hielt sich eine andauernde Renitenz der alten Unterschichten gegen die Repressionen von Militär und Verwaltung. Das Mißtrauen war auf beiden Seiten tief verwurzelt. Am Kaisergeburtstag vermieden es die Einheimischen, ihre Häuser zu verlassen, und noch lange Zeit hegten sie die ernsthafte Hoffnung auf rasche Rückeroberung des Landes ${ }^{46}$. Schließlich, und damit sei nochmals der Bogen zum Kriegsschauplatz vom Herbst 1870 gespannt, konnte die verbliebene französische Bevölkerung nur schwer vergessen, wie stark sie in den Monaten der Belagerung unter den deutschen Bundesheeren gelitten hatte. Elsaß-Lothringen jeden-

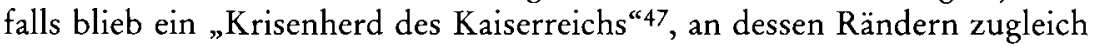
die Temperatur der politischen Beziehungen zwischen Deutschland und Frankreich wie an einem Thermometer abzulesen waren.

Was ist übrig von den Schlachten und der Belagerung um Metz 1870? Neben den Denkmälern bietet die Gedenkstätte in Gravelotte - vergleichbar Cospeda bei Jena, wo in einer ebensolchen liebevoll-antiquarischen, aber weit weniger nationalbewußten Art und Weise an die dortige Schlacht vom Oktober 1806 erinnert wird - viel Anschauliches, eben mit Sorgfalt und kaum unter kritischen, auch die Rezeption einschließenden Gesichtspunkten Zusammengetragenes. Eine tranchée des baïonnettes ${ }^{48}$, wie in Verdun, findet man hier wie dort nicht, und fast scheint es, als könne dem Krieg vor 1914 noch Spielerisches und Anmutiges, ja sogar Heldenhaftes abgewonnen werden. Wenn die vorliegende Studie dies auch widerlegten konnte, so war der Kampf um die Festung Metz nichtsdestoweniger von vormodernen Zügen geprägt. Doch ist dessen Geschichte durchaus kein Fall für die Antiquitätenkiste. Der Charakter des maschinellen Krieges der Zukunft deutete sich an und wies auf den Übergang vom „Treffen im freien Feld“ und vom „Duell beweglicher Heere“ hin zum Stellungskrieg und zum „Kampf der Mörser“. Im kleinen bewegen uns noch immer Fragen wie jene nach dem Verhältnis von Frauen zum Militär oder der Art und Weise, wie man Grenzen sichern

44 Verfassung mit Zweikammersystem, wovon die zweite Kammer durch allgemeines, gleiches, direktes und geheimes Wahlrecht gewählt wurde.

45 Zit. nach BRONNER, 1870/71, S. 227.

46 STÄHLIN, Geschichte Elsaß-Lothringens, S. $215 \mathrm{f}$.

47 WEHLER, Verfassungsreform.

48 Laufgraben, in dem eine Abteilung Gewehr bei Fuß stehend verschüttet wurde und nur noch die Bajonettspitzen aus der Erde ragen. 
soll. Auch der freilich zu einem guten Teil der Besonderheit des Festungsstandortes geschuldete Umstand, daß der Krieg vor Metz gnadenlos in den zivilen Sektor ausgriff, nahm Künftiges vorweg. Wie hier der Wandel Kontinuitäten zu überlagern begann, so gewannen auch Feindbilder und Nationalismen eine neue Qualität, deren lange Schatten auf den Kulturkrieg von 1914 weisen. Noch immer und nun neuerlich zugespitzt glaubten sich Deutsche und Franzosen im ewigem Geschwisterzwist, und einer tausendjährigen Feindschaft wurde diesseits und jenseits des Rheins das Wort geredet. So geriet das „Zwischenreich“ oder Grenzland hier zum Angelhaken einer revanchelüsternen Stimmung, dort zum unantastbaren Landstrich, an den sich "hohe nationale Werte" 49 banden. Es ist die Frage, ob ein „französisches Metz" und ein deutscher Verzicht etwa auf ganz Lothringen daran etwas geändert hätten. Im kollektiven Gedächtnis hielten sich, als Symbol des Triumphes hier und des Verlusts dort, eher Sedan und Straßburg als das langwierige und zähe Ringen um Stadt und Festung. Auf lange Sicht entwickelte sich im emotional aufgeladenen Grenzraum ein zwar verschiedentlich begründeter, aber letztlich doch ähnlich militärgläubig-folkloristischer Totenkult, der beide Nationen gleichermaßen umtrieb. Die Franzosen erinnerten sich darüber hinaus noch eine Zeitlang mit Bitterkeit an Bazaine und das „il a fumé la pipe avec les Allemands“, und der ridiküle Schauprozeß gegen den kaiserlichen Marschall sollte der verletzten nationalen Seele einen Sündenbock präsentieren und das militärische wie politische Desaster des gesamten Krieges verdrängen. Zumindest dadurch blieb die öffentliche Aufmerksamkeit dann doch noch ein wenig auf Metz fixiert. Daß den schlimmsten Kapiteln deutsch-französischer Beziehungen damit insgesamt der Boden bereitet war, ahnten indes die Wenigsten.

Erst die Erfahrung zweier Weltkriege brach mit traditionellen Mustern: An die Stelle unabänderlicher Feindschaft trat konkurrierende Akzeptanz, und aus feindseligem Gegeneinander wurde verantwortliches Miteinander. In der europäischen Friedensordnung verloren Waffenplätze wie Metz an Bedeutung, fast ebenso wie einst durch die pax romana die attischen Festungen, die über Jahrhunderte strategisch bedeutsame Bastionen in der Auseinandersetzung mit Persern, Spartanern oder Makedoniern gewesen waren ${ }^{50}$. Doch denkt man weiterhin zumindest in Kategorien wie jener von der $\mathrm{Fe}$ stung Europa, gleichwohl in gänzlich anderer Bedeutung als der ursprünglichen. Nicht mehr einer militärischen, sondern einer zivilen Invasion aus Süd und Ost gilt es nunmehr zu widerstehen. Auch drängt der vergleichende Blick zur Überlegung, ob im zu Ende gegangenen Ost-West-Konflikt die berüchtigten Mauerschützen - gleichwohl hier nur von einer Seite geschossen

49 Cartellieri, Deutschland und Frankreich, S. 15.

50 Die Bretterwände starrer Perioden überbrückend, wäre hier etwa der Vergleich zwischen Metz und dem griechischen Rhamnos reizvoll. Vgl. Poulllpux, Forteresse. Mögliche theoretische Ansätze bei KAELBLE, Vergleich. 
wurde - nicht auch Vorposten in einem großen kalten Belagerungskrieg waren.

Die alten Festungen jedenfalls erscheinen uns heute wie unbrauchbar gewordene Vehikel dynastischer und nationaler Abschottung, wie Attribute einer sich verpuppenden und verpanzernden Selbstfindung, die schließlich explosiv wurde. Das Grenzland mit seinen Schlachtfeldern um Metz ist inzwischen zum Bindeglied einer europäischen Region gewachsen, der europäischen Region vielleicht. Allerdings bleibt auch aus heutiger Sicht bereits mit Blick auf die Peripherie des Kontinents und mehr noch auf den Nahen und Mittleren Osten das generelle Phänomen der Feindschaft und des Krieges sehr wohl bestehen - eines Krieges, der, so scheint es, nicht mehr erklärt wird und sich zuletzt in Gestalt des Hightech-Terrorismus fanatischer Fundamentalisten immer mehr, ja fast ausschließlich gegen Zivilisten richtet. 